\title{
Community acquired respiratory virus infections in adult patients undergoing umbilical cord blood transplantation
}

\author{
Juan Montoro ${ }^{1}$ Jaime Sanz $\mathbb{E}^{1,2} \cdot$ Ignacio Lorenzo $^{1}$ - Aitana Balaguer-Roselló ${ }^{1}$ Miguel Salavert ${ }^{3}$. \\ María Dolores Gómez ${ }^{4}$ Manuel Guerreiro (1) ${ }^{1}$ - Eva M. González Barberá ${ }^{4}$. Cristina Aguado ${ }^{5}$ - Luiza Tofán ${ }^{5}$. \\ Guillermo F. Sanz $\mathbb{1}^{1,2} \cdot$ Miguel A. Sanz ${ }^{1,2,6} \cdot$ José Luis Piñana $\mathbb{C}^{1,2}$
}

Received: 2 March 2020 / Revised: 28 April 2020 / Accepted: 1 May 2020 / Published online: 15 May 2020

(c) The Author(s), under exclusive licence to Springer Nature Limited 2020

\begin{abstract}
Characteristics and risk factors (RFs) of community-acquired respiratory virus (CARV) infections after umbilical cord blood transplantation (UCBT) are lacking. We retrospectively analyzed CARV infections in 216 single-unit myeloablative UCBT recipients. One-hundred and fourteen episodes of CARV infections were diagnosed in $62(29 \%)$ patients. Upper respiratory tract disease (URTD) occurred in 61 (54\%) whereas lower respiratory tract disease (LRTD) in 53 (46\%). The 5-year cumulative incidence of CARV infection was $29 \%$. RFs for developing CARV infections were: prednisone-based graft-versus-host disease (GVHD) prophylaxis and grade II-IV acute GVHD. RFs analysis of CARV progression to LRTD identified 2007-2009 period and absolute lymphocyte count (ALC) $<0.5 \times 10^{9} / \mathrm{L}$. ALC $<0.5 \times 10^{9} / \mathrm{L}$ had a negative impact on day 60 mortality in both overall CARV and those with LRTD, whereas proven LRTD was associated with higher day 60 mortality. CARV infections had a negative effect on non-relapse mortality. Overall survival at day 60 after CARV detection was significantly lower in recipients with LRTD compared with URTD (74\% vs. 93\%, respectively). In conclusion, CARV infections after UCBT are frequent and may have a negative effect in the outcomes, in particular in the context of lymphocytopenia.
\end{abstract}

\section{Introduction}

Upper and/or lower respiratory tract disease (URTD/LRTD) due to community-acquired respiratory viruses (CARVs) represent a significant cause of morbidity and mortality in recipients of

Supplementary information The online version of this article (https:// doi.org/10.1038/s41409-020-0943-0) contains supplementary material, which is available to authorized users.

José Luis Piñana

jlpinana@gmail.com

1 Department of Hematology, University Hospital La Fe, Valencia, Spain

2 CIBERONC, Instituto Carlos III, Madrid, Spain

3 Department of Infectious Diseases, University Hospital La Fe, Valencia, Spain

4 Department of Microbiology, University Hospital La Fe, Valencia, Spain

5 Department of Laboratory Medicine, University Hospital La Fe, Valencia, Spain

6 Department of Medicine, University of Valencia, Valencia, Spain allogeneic hematopoietic stem cell transplantation (allo-HSCT) [1-8]. Decreased absolute lymphocyte count (ALC) at the time of CARV infection is probably the main negative prognostic factor for progression to LRTD and is associated with an increased mortality after allo-HSCT $[6,7,9,10]$. With this in mind, we can hypothesize that CARV infections in the umbilical cord blood transplantation (UCBT) setting, which is associated with delayed T-cell immune reconstitution, could be associated with greater morbidity and mortality than with other modalities of allo-HSCT. However, data regarding CARV infections after UCBT are lacking.

The aim of this study was to analyze the incidence of CARV infections, causative viruses, clinical characteristics, risk factors (RFs), and outcomes in a large series of adult patients undergoing UCBT.

\section{Methods}

\section{Patients}

Between January 2007 and January 2019, 216 consecutive adult patients with hematologic malignancies 
who underwent myeloablative UCBT at our institution were included in this study. Eligibility criteria for UCBT and UCB selection have been extensively described elsewhere [11-13]. The institutional review board approved the clinical protocols. Written informed consent was obtained from all patients according to the declaration of Helsinki.

\section{Conditioning regimen and graft-versus-host disease prophylaxis}

Myeloablative conditioning regimen consisted of thiotepa, busulfan, and fludarabine. Rabbit antithymocyte globulin (ATG) was also given, except for patients enrolled in clinical trials $\mathrm{NiCord}^{\circledR}$ or StemEx ${ }^{\circledR}(n=14)$. Graft-versushost disease (GVHD) prophylaxis consisted of cyclosporine combined with mycophenolate mofetil or a short course of prednisone [14].

\section{CARV infections management}

From 2007 to April 2016, patients with influenza virus infection were treated with oseltamivir $75 \mathrm{mg} / 12 \mathrm{~h}$ orally for 5 days. Recipients with persistent and severe respiratory symptoms and repeated influenza virus detection received a second course of oseltamivir. For recipients with respiratory syncytial virus (RSV) infection, only those with proven LRTD were treated with aerosolized ribavirin $(2 \mathrm{~g} / 8 \mathrm{~h})$ until improvement. Patients with human parainfluenza virus (HPiV) infection were not routinely treated with ribavirin, except in cases with respiratory failure and oxygen support. From May 2016, oseltamivir $75 \mathrm{mg} / 12 \mathrm{~h}$ orally was given to recipients with influenza virus infection until resolution symptoms. In cases of severe pneumonia with oxygen requirements and/or prolonged shedding with persistent respiratory symptoms ( $>3$ weeks) an increased oseltamivir dose $(150 \mathrm{mg} / 12 \mathrm{~h})$ was given until resolution of respiratory symptoms. RSV and HPiV were managed according to our interventional protocol [15]. Briefly, ribavirin therapy was instituted in recipients with LRTD caused by RSV or HPIV (aerosolized formulation), whereas recipients with URTD (oral formulation) had to score $\geq 3$ points of the immunodeficiency scoring index (ISI) points and/or $\geq 2 \mathrm{RFs}$ according to the ECIL-4 guidelines [16], and/or present one or more co-infective virus(es) before starting ribavirin. Annual influenza vaccination was recommended to all patients after the third month following UCBT, except for patients with moderate to severe GVHD at the time of vaccination program who had received gammaglobulin, ATG or rituximab within the 3 months before the flu vaccine period. In these patients, vaccination was delayed at physician discretion [17].

\section{Definitions}

According to the ECIL-4 recommendations [16], URTD was defined by the combination of upper respiratory symptoms along with identification of a CARV by microbiological test, and the absence of LRTDs symptoms and/or any indication of pulmonary infiltrates by chest X-ray or computed tomography scan. LRTD was classified as possible or confirmed as previously described [18]. Possible LRTD was defined by the detection of a CARV in the upper respiratory tract in recipients developing new pulmonary infiltrates. Confirmed LRTD was defined when the above-mentioned clinical features were accompanied by isolation of the virus in tracheal aspirates or by bronchoalveolar lavage (BAL). Probable LRTD was not considered, since bronchoscopies were not performed in patients without radiological proof of pulmonary involvement. Progression of a URTD to an LRTD was defined as the onset of pneumonia in patients with a prior or concurrent URTD [10].

Respiratory co-infection was defined as the identification of significant microbiological agents in the same sample as previously defined [7].

\section{Technical and diagnostic considerations}

All patients who had received an UCBT and had signs and symptoms of URTD or LRTD proceed to virologic evaluation. From January 2007 to December 2009, the CARV screening strategy relied on rapid detection test such as shell vial cultures (Vircell, Spain), direct staining by using monoclonal antibodies for the presumptive detection of RSV, influenza A and B virus, HPiV 1, 2, and 3 and adenovirus (ADV) (DAKO, USA) or by using an in vitro rapid immunochromatographic assay that detected RSV and Influenza A and B (Alere BinaxNOW ${ }^{\circledR}$ ). From January 2010 to April 2016, first-line CARV screening test was based on rapid antigen detection for influenza and RSV through the automated Simplexa ${ }^{\mathrm{TM}}$ influenza A/B and VRS Direct assay (SPX, Focus Diagnostics). Patients with negative results in rapid antigen detection with impaired respiratory symptoms or requiring hospital admission underwent a second-line test based on a RT-PCR multiplex platform consisting on the CLART ${ }^{\circledR}$ PneumoVir DNA array assay (Genomica, Spain). This RT-PCR method can detect ADVs; human bocavirus (HBoV); human coronavirus (HCoV) types 229E; influenza A virus A/H1N1, A/H3N2; influenza $\mathrm{B}$ and $\mathrm{C}$; human metapneumovirus (HMPV); HPiV 1, 2, 3, and 4; RSV A-B; and enterovirus/rhinovirus (EvRh). From May 2016, first-line CARV screening was performed by the CLART $^{\circledast}$ PneumoVir DNA array assay and from July 2018 by BioFire FilmArray ${ }^{\circledR}$ Respiratory Panel (BioFire Diagnostics, Salt Lake City). This RT-PCR assay is able to detect 15 respiratory viruses: influenza virus 
types A, B, and C, ADV, HCoV HKU1, NL63, 229E and OC43, HMPV, EvRh, HPiV types 1-4 and RSV, as well as three bacteria: Mycoplasma pneumoniae, Chlamydia pneumoniae, and Bordetella pertussis.

\section{Statistical analysis}

The primary endpoint was to determine the incidence of CARV infection as well as RFs for CARV progression to LRTD. Secondary endpoints were to identify RFs for developing CARV infections and its effect on non-relapse mortality (NRM) and all-cause mortality and overall survival (OS) at day 60 after the identification of CARV infection.

For cumulative incidence of CARV infection and its impact on the NRM after UCBT we accounted for the first CARV episode, whereas for RFs analyses of CARV LRTD progression and day 60 all-cause mortality and OS we include all CARV episodes that occurred in recipients who were relapse-free at the time of CARV detection. The probabilities of posttransplant events such as CARV infection, acute and chronic GVHD, engraftment, NRM, and relapse were estimated by the cumulative incidence method [19, 20]. Univariate analyses of posttransplantation outcomes were calculated using the Gray test [21]. For cumulative incidence analyses of CARV infection, death in complete remission and relapse occurring before CARV were considered as competing events, while relapse was the competing event for NRM. The assumption of proportional hazards over time was tested for all posttransplant explanatory covariates using a time-dependent covariate by univariate Cox regression model. When a time-dependent covariate was included in the final models, multivariate analyses were carried out by Cox proportional hazards regression with inclusion of those variables with a $p<0.1$ in the prior univariate testing. To assess the impact of GVHD and CARV infection in UCBT outcomes (NRM and OS), these variables were analyzed as time-dependent covariates.

Univariate and multivariate analyses of the association of clinical and laboratory RFs with progression of CARV infection to LRTD, CARV infection day 60 mortality, and LRTD CARV day 60 mortality were calculated using logistic regression models. If odds ratio could not be generated because one group had zero events and the maximum likelihood estimation of the coefficient was infinity, the Haldane's correction was applied [22]. For multivariate analysis, only variables with parameter estimates showing a $p \leq 0.10$ in the univariate analysis were finally included. Two-sided exact $P$ values were reported and $p \leq 0.05$ were considered statistically significant. The probability of OS was estimated from the time of CARV detection using Kaplan-Meier curves [23]. All statistical analyses were performed using SPSS and R.

\section{Results}

\section{Patient, disease, and transplant characteristics}

The main patient, disease, and transplant characteristics are shown in Table 1. Briefly, the median age at time of transplantation was 38 years and $127(59 \%)$ were males. Most of recipients had acute leukemia and myelodysplastic syndrome (84\%). One-hundred twenty-five patients $(58 \%)$ were transplanted in early phase. The majority of patients (91\%) received ATG as a part of conditioning regimen. GVHD prophylaxis with cyclosporine and prednisone was used in $57 \%$ of patients. Except for six patients $(3 \%)$ who received a fully matched $6 / 6 \mathrm{UCB}$ unit, the remaining patients received a partially matched graft.

\section{Posttransplant outcomes}

One hundred ninety patients (88\%) achieved neutrophil engraftment at a median time of 20 days. The cumulative incidence of grade II-IV acute GVHD (aGVHD) at 100 days was $45 \%$. The 5 -year cumulative incidence of overall chronic GVHD, relapse, NRM, and OS was 64\%, $30 \%, 46 \%$, and $23 \%$, respectively.

\section{Characteristics of CARV infections}

Details of episodes of CARV infections are shown in Supplementary Table 1 . A total of 114 CARV infections were observed in $62(29 \%)$ recipients at a median time of 253 days (range, -1 to 2902) after stem cell infusion. Thirty-one (14\%) recipients had one CARV episode whereas $31(14 \%)$ had two or more episodes. Sixty-one (54\%) episodes of CARV infections were limited to the URTD whereas $53(46 \%)$ had LRTD involvement. The most common type of CARV identified was RSV in 46 (34\%) cases, followed by EvRh in $32(23 \%)$, and influenza in $31(23 \%)$. As shown in Fig. 1, CARV infections predominated in the winter months (84 episodes, 74\%).

The overall 5-year cumulative incidence of CARVs was 29\% (95\% confidence interval (C.I.), 22-35\%) (Fig. 2a). The 1-year cumulative incidence according to the study period was $10 \%$ (95\% C.I., 4-17\%) from 2007 to 2009, $28 \%$ (95\% C.I., 20-36\%) from 2010 to April 2009, and $44 \%$ (95\% C.I., $21-67 \%$ ) the remaining period, respectively $(p<0.001)$ (Fig. 2b).

\section{Risk factors for CARV infection and NRM}

Univariate and multivariate analyses of RFs for NRM and development of CARV infection are shown in Table 2. Multivariate analysis identified prednisone-based GVHD prophylaxis (Hazard Ratio [HR] 2.3, 95\% C.I., 1.3-4, $p=$ 
Table 1 Patients, graft- and transplantation related characteristics and outcomes.

\begin{tabular}{|c|c|}
\hline Characteristic & Value \\
\hline No. of patients & 216 \\
\hline Age, median (range) & $38(15-59)$ \\
\hline Male recipient, $n(\%)$ & $127(59)$ \\
\hline \multicolumn{2}{|l|}{ Diagnosis, $n(\%)$} \\
\hline AL/MDS & $182(84)$ \\
\hline Chronic myeloid leukemia & $7(3)$ \\
\hline Chronic lymphoproliferative disorders & $19(9)$ \\
\hline Other & $8(4)$ \\
\hline \multicolumn{2}{|l|}{ Disease status at transplant, $n(\%)$} \\
\hline Early & $125(58)$ \\
\hline Intermediate & $53(24)$ \\
\hline Advances & $38(18)$ \\
\hline Prior ASCT, $n(\%)$ & $16(7)$ \\
\hline \multicolumn{2}{|l|}{ HLA compatibility, $n(\%)$} \\
\hline 6 of 6 & $6(3)$ \\
\hline 5 of 6 & $36(16)$ \\
\hline 4 of 6 & $170(79)$ \\
\hline 3 of 6 & $4(2)$ \\
\hline $\begin{array}{l}\text { Recipient CMV positive serological status before } \\
\text { transplantation, } n(\%)\end{array}$ & $164(76)$ \\
\hline Female donor to male recipient, $n(\%)$ & $66(30)$ \\
\hline \multicolumn{2}{|l|}{ Conditioning regimen, $n(\%)$} \\
\hline $\mathrm{TT}+\mathrm{BU}+\mathrm{FLU}+\mathrm{ATG}$ & $197(91)$ \\
\hline $\mathrm{TT}+\mathrm{BU}+\mathrm{FLU}$ & $19(9)$ \\
\hline \multicolumn{2}{|l|}{ GVHD prophylaxis, $n(\%)$} \\
\hline Cyclosporine $\mathrm{A}+$ prednisone & $122(57)$ \\
\hline Cyclosporine A + MMF & $94(43)$ \\
\hline Median no. of $\mathrm{CD} 34^{+}$cells infused, $\times 10^{5} / \mathrm{kg}$ (range) & $1.6\left(0.4-21.5^{\mathrm{a}}\right)$ \\
\hline Median no. of nucleated cells infused, $\times 10^{7} / \mathrm{kg}$ (range) & $2.8(1.1-7.2)$ \\
\hline \multicolumn{2}{|l|}{ Transplant outcomes } \\
\hline Neutrophil engraftment, $n(\%)$ & $190(88)$ \\
\hline Primary engraftment failure & $12(5)$ \\
\hline Early death before engraftment & $10(4)$ \\
\hline \multicolumn{2}{|l|}{ Median days to myeloid recovery, days (range) } \\
\hline Neutrophils $>0.5 \times 10^{9} / 1$ & $20(7-55)$ \\
\hline \multicolumn{2}{|l|}{ Acute GVHD } \\
\hline Cum. Inc. of aGVHD II-IV at 100 days, \% (95\% C.I.) & $45(38-52)$ \\
\hline Median onset, days (range) & $27(4-124)$ \\
\hline Cum. Inc. of aGVHD III-IV at 100 days, \% (95\% C.I.) & $17(12-22)$ \\
\hline \multicolumn{2}{|l|}{ Chronic GVHD } \\
\hline Cum. Inc. of cGHVD at 5 year, $\%$ (95\% C.I.) & $64(57-71)$ \\
\hline Median onset in days (range) & $145(70-702)$ \\
\hline Cum. Inc. of cGHVD Ext, \% (95\% C.I.) & $41(34-49)$ \\
\hline \multicolumn{2}{|l|}{ NRM, \% (95\% C.I.) } \\
\hline At day +100 & $15(10-20)$ \\
\hline At 1 year & $38(32-45)$ \\
\hline At 5 years & $46(40-53)$ \\
\hline OS at 5 years, $\%$ (95\% C.I.) & $23(17-29)$ \\
\hline Median follow-up for survivors, days (range) & $2558(139-4526)$ \\
\hline
\end{tabular}

$A L$ acute leukemia, $M D S$ myelodysplastic syndrome, $A S C T$ autologous stem cell transplant, $T T$ thiotepa, $B u$ busulfan, $A T G$ anti-thymoglobuline, $F U$ fludarabine, $M M F$ mycophenolate mofetil, Cum. Inc cumulate incidence, C.I. confident interval, GVHD graft-versus-host disease, $N R M$ non-relapse mortality, $O S$ overall survival.

${ }^{\mathrm{a}} \mathrm{A}$ patient enrolled in an ex vivo expanded umbilical cord blood protocol.

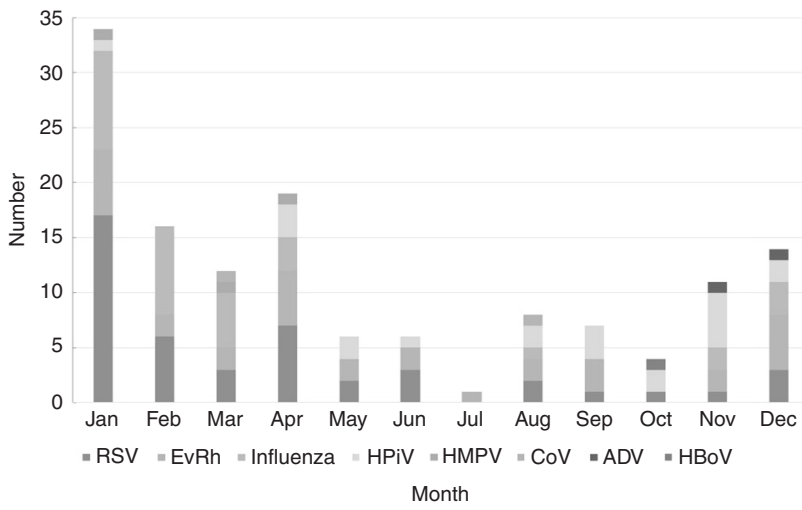

Fig. 1 CARV infections. Type of CARV infection according to the month of detection.

0.003) and grade II-IV aGVHD (HR 2.4, 95\% C.I., 1.4-4.1, $p=0.001)$ as the only independent variables.

Regarding NRM, the variables associated with increased mortality were: $\geq 1$ previous autologous HSCT (HR 2.1, 95\% C.I., 1.1-3.8, $p=0.01$ ), prednisone-based GVHD prophylaxis (HR 1.5, 95\% C.I., $1-2.3, p=0.05$ ), grade II-IV aGVHD (HR 1.7, 95\% C.I., 1.1-2.6, $p=0.01$ ), and development of CARV infection (HR 1.8, 95\% C.I., $1.1-2.9, p=0.009$ ).

\section{Risk factors for progression to LRTD and for day 60 mortality}

Univariate and multivariate analyses of RFs for CARV infection progression to LRTD and for day 60 mortality are shown in Table 3. The following two independent conditions were associated with progression to LRTD: 2007-2009 period (OR 8, 95\% C.I., 1.7-37.8, $p=0.008$ ) and $\mathrm{ALC}<0.5 \times 10^{9} / \mathrm{L}$ (OR 3, 95\% C.I., $1.1-8.9, p=0.03$ ). Multivariate analysis also identified ALC $<0.5 \times 10^{9} / \mathrm{L}(\mathrm{OR}$ 8.4, 95\% C.I., $2.2-32.4, p=0.002)$ and proven RVI LRTD (OR 4, 95\% C.I., 1.3-12.7, $p=0.016$ ) as independent factors associated with day 60 mortality. A subanalysis of 60 day mortality in patients with LRTD due to CARV only identified ALC $<0.5 \times 10^{9} / \mathrm{L}$ as independent factor (OR $15.2,95 \%$ C.I., $1.7-132, p=0.013)$.

\section{Causes of mortality and overall survival by day $\mathbf{6 0}$ after CARV infection}

Crude 60-day mortality after CARV infection was $16 \%$, with a median time to death of 26 days (range 6-53 days). Causes of mortality were respiratory failure attributable to the LRTD in 13 cases, two GVHD, one septic shock, one hematological relapse, and one intracranial hemorrhage. Differences in mortality according to the type of virus involved were not statistically significant (data not shown). 


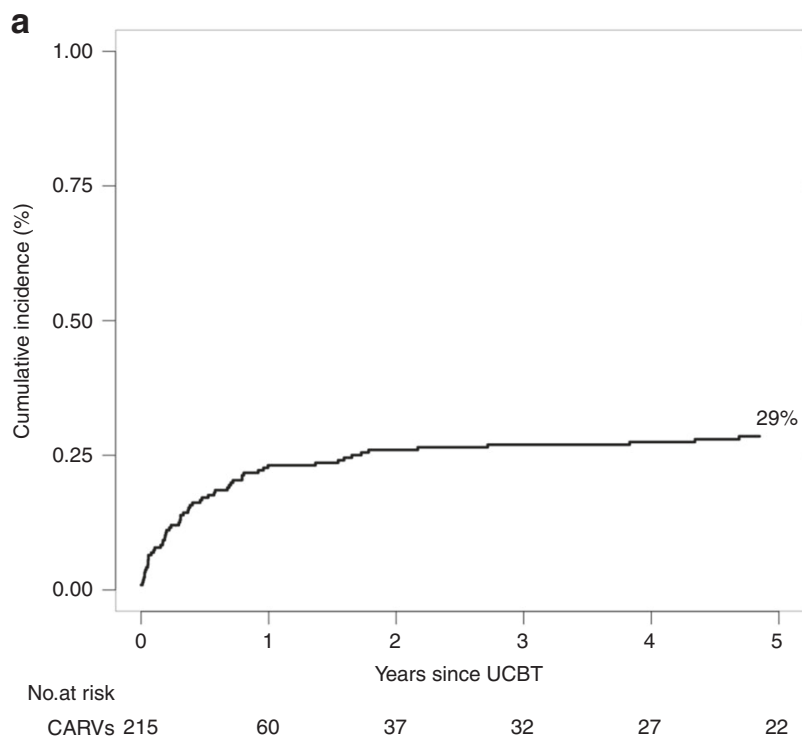

b

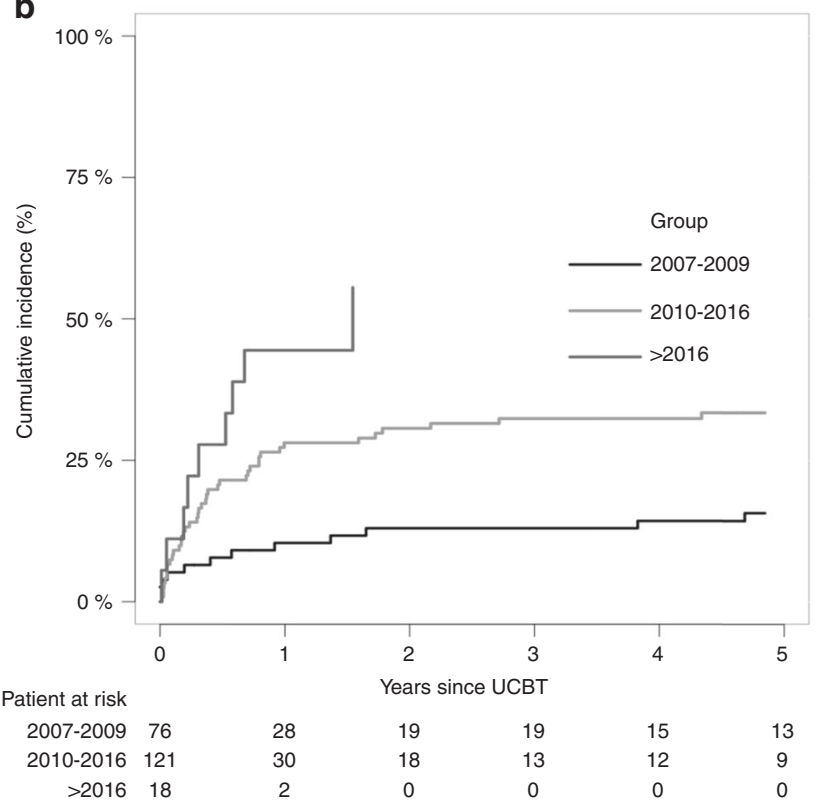

Fig. 2 Cumulative incidence of CARV. a Cumulative incidence of CARV infection after UCBT in the entire cohort. b Cumulative incidence of CARV infection according to the UCBT period.

Day 60 OS for patients with URTD and LRTD was 93\% and $74 \%$, respectively $(p=0.004)$ and $96 \%$ for LRTD CARV with ALC $>0.5 \times 10^{9}$ vs. $55 \%$ for those with ALC $\leq$ $0.5 \times 10^{9}(p=0.001)$ (Fig. 3a, b).

\section{Discussion}

This study shows that CARV infections are common and represent a significant problem after myeloablative single-unit UCBT. We identified two independent RFs that significantly increased the susceptibility to CARV infections: the use of prednisone for prevention of GVHD and the development of grade II-IV acute GVHD. Our data supports that CARV infections had a negative effect on NRM after UCBT. Lymphopenia significantly increased the risk of progression to LRTD and both overall CARV and LRTD mortality. Proven RVI LRTD was also associated with higher mortality.

We found a CARV infection incidence of $29 \%$ in UCBT recipients, which is comparable to those reported in other series with other stem cell sources [10, 24]. In contrast, our incidence seems significantly higher when compared with studies conducted before the multiplex PCR era [6, 25]. In fact, we observed in our series that the CARV incidence increased as we introduced more sophisticate and sensitive CARVs screening tests $(10 \%$ with rapid cultures, $28 \%$ with rapid antigen detection technics, and $44 \%$ with multiplex PCR platforms, Fig. 2b). This observation emphasizes that efforts in screening CARVs with highly sensitive technics are required to better understand the real epidemiology and incidence of CARV infections after UCTB.

The most common identified CARV causative agent was RSV followed by EvRh, influenza, and HPiV. These relatives incidences are most probably a direct consequence of the use of several CARV screening test in different periods with different sensitivities and should not be used as a realistic epidemiological data. As we previously reported, $\mathrm{EvRh}$ is the most common circulating CARV in the alloHSCT setting when multiplex PCR platforms are used [8]. In contrast, we provided seasonal data which is consistent with prior reports $[8,26]$ supporting that most of CARV infections after UCBT occurred during the cold months (from October to May).

We identified the use of prednisone as GVHD prophylaxis and developing grade II-IV aGVHD as the main RFs for increased susceptibility to develop symptomatic CARV infection in UCBT recipients. These findings are not surprising since it is well-known that corticosteroids and moderate to severe GVHD increased the risk and severity of viral infection [27].

Importantly, near a half of UCBT recipients with CARV infection progress to LRTD supporting the profound immunosuppression status of UCBT recipients. Because $79 \%$ of our UCBT recipients who develop LRTD undergo BAL examination, the observed results of LRTDs by CARVs are probably an accurate measure of the true frequency of these infections. We identified lymphopenia $<0.5 \times 10^{9} / \mathrm{L}$ as risk factor for progression to LRTD. This finding is somewhat expected since lymphopenia was the main risk factor for LRTD progression in several studies $[6,7,9,10]$. Another relevant finding was that the earlier period of UCBT (2007-2009) was associated with higher probability of LRTD progression. This data suggest that the progressive introduction of more sophisticate CARVs screening technics could lead not only to an increased 
Table 2 Risk factors for CARVs development and NRM: univariate and multivariate analyses.

\begin{tabular}{|c|c|c|c|c|c|c|c|c|}
\hline \multirow[t]{3}{*}{ Variables } & \multicolumn{4}{|l|}{$\begin{array}{l}\text { CARV } \\
(n=216)\end{array}$} & \multicolumn{4}{|l|}{$\begin{array}{l}\text { NRM } \\
(n=216)\end{array}$} \\
\hline & \multicolumn{2}{|l|}{$\begin{array}{l}\text { Fine and Gray test } \\
\text { (Univariate analysis) }\end{array}$} & \multicolumn{2}{|l|}{ Cox Regr. } & \multicolumn{2}{|l|}{ Univariate analysis } & \multicolumn{2}{|l|}{ Cox Regr. } \\
\hline & CI $(95 \%$ confidence interval $)$ & $P$ & HR & $P$ & CI (95\% confidence interval) & $P$ & HR & $P$ \\
\hline \multicolumn{9}{|l|}{ Recipient age, years } \\
\hline$\leq 38$ & $25(17-33)$ & 0.1 & & & $42(33-51)$ & 0.3 & & \\
\hline$>38$ & $34(24-43)$ & & & & $51(41-61)$ & & & \\
\hline \multicolumn{9}{|l|}{ Diagnosis } \\
\hline $\mathrm{AL}$ & $28(22-35)$ & 0.6 & & & $46(39-54)$ & 0.9 & & \\
\hline Others & $37(17-56)$ & & & & $46(27-65)$ & & & \\
\hline \multicolumn{9}{|c|}{ Diagnosis status at transplant } \\
\hline Early & $32(24-41)$ & 0.2 & & & $44(36-53)$ & 0.4 & & \\
\hline Others & $25(16-34)$ & & & & $49(39-60)$ & & & \\
\hline \multicolumn{9}{|l|}{ Prior ASCT } \\
\hline No & $24(19-30)$ & 0.9 & & & $43(36-50)$ & $<0.001$ & $2.1(1.1-3.8)$ & 0.01 \\
\hline Yes & $25(4-46)$ & & & & 100 & & & \\
\hline \multicolumn{9}{|l|}{ HLA compatibility } \\
\hline $4 / 6$ & $28(22-35)$ & 0.7 & & & $47(39-54)$ & 0.9 & & \\
\hline$>4 / 6$ & $34(19-49)$ & & & & $45(30-60)$ & & & \\
\hline \multicolumn{9}{|c|}{ ATG as part of conditioning } \\
\hline No & $26(7-46)$ & 0.9 & & & $29(8-51)$ & 0.1 & & \\
\hline Yes & $28(21-34)$ & & & & $48(41-55)$ & & & \\
\hline \multicolumn{9}{|c|}{ Infused CD34,$+ \times 10^{5} / \mathrm{kg}$} \\
\hline$<1.6$ & $25(17-34)$ & 0.3 & & & $46(36-55)$ & 0.8 & & \\
\hline$\geq 1.6$ & $31(23-43)$ & & & & $47(38-57)$ & & & \\
\hline \multicolumn{9}{|l|}{ Infused $\mathrm{TNC}, \times 10^{7} / \mathrm{kg}$} \\
\hline$<2.8$ & $24(16-32)$ & 0.1 & & & $48(38-57)$ & 0.7 & & \\
\hline$\geq 2.8$ & $33(24-42)$ & & & & $45(36-55)$ & & & \\
\hline \multicolumn{9}{|l|}{ GVHD prophylaxis } \\
\hline PDN & $35(26-43)$ & 0.02 & $2.3(1.3-4)$ & 0.003 & $52(43-61)$ & 0.08 & $1.5(1-2.3)$ & 0.05 \\
\hline MMF & $22(13-30)$ & & & & $39(29-49)$ & & & \\
\hline aGVHD grade II-IV ${ }^{\mathrm{a}}$ & $1.7(1.1-2.7)$ & 0.008 & $2.4(1.4-4.1)$ & 0.001 & $3(2.2-4.1)$ & $<0.001$ & $1.7(1.1-2.6)$ & 0.01 \\
\hline aGVHD grade III-IV ${ }^{\mathrm{a}}$ & $1(0.6-1.6)$ & 0.9 & & & $2.8(2.2-3.6)$ & $<0.001$ & NT & \\
\hline CARV & & & & & $3.4(2.3-5)$ & $<0.001$ & $1.8(1.1-2.9)$ & 0.009 \\
\hline
\end{tabular}

Results are expressed as HR from univariate COX. Regr model.

$A L$ acute leukemia, $P D N$ prednisone, $M M F$ mycophenolate mofetil, GVHD graft-versus-host disease, $A S C T$ autologous stem cell transplant, $A T G$ anti-thymoglobuline, $N T$ not tested in the multivariate, since grade III-IV acute GVHD is a subcategory of grade II-IV acute GVHD.

${ }^{a}$ Analyzed as time-dependent covariates.

ability in detecting CARVs (and as a consequence observing a higher incidence of CARV) but more importantly that the use of less sensitive test may have produced a delay in the identification of CARVs in the URTD stage an thus a delay in the inception of antiviral drugs when available before the progression to LRTD. Finally, it is of interest to highlight that in approximately one third of the cases copathogens were detected, being striking the frequent co-pathogenicity with at least one respiratory virus, as also previously reported for other stem cell sources [8, 24].

We analyzed the consequence of CARV infections on NRM in our cohort of UCBT recipients, identifying infections as the primary cause of NRM, which is line with most reports that analyzed mortality in UCBT [28, 29]. Our multivariate analysis showed an independent negative effect of CARV infections on NRM. Again, this finding supports that CARV 


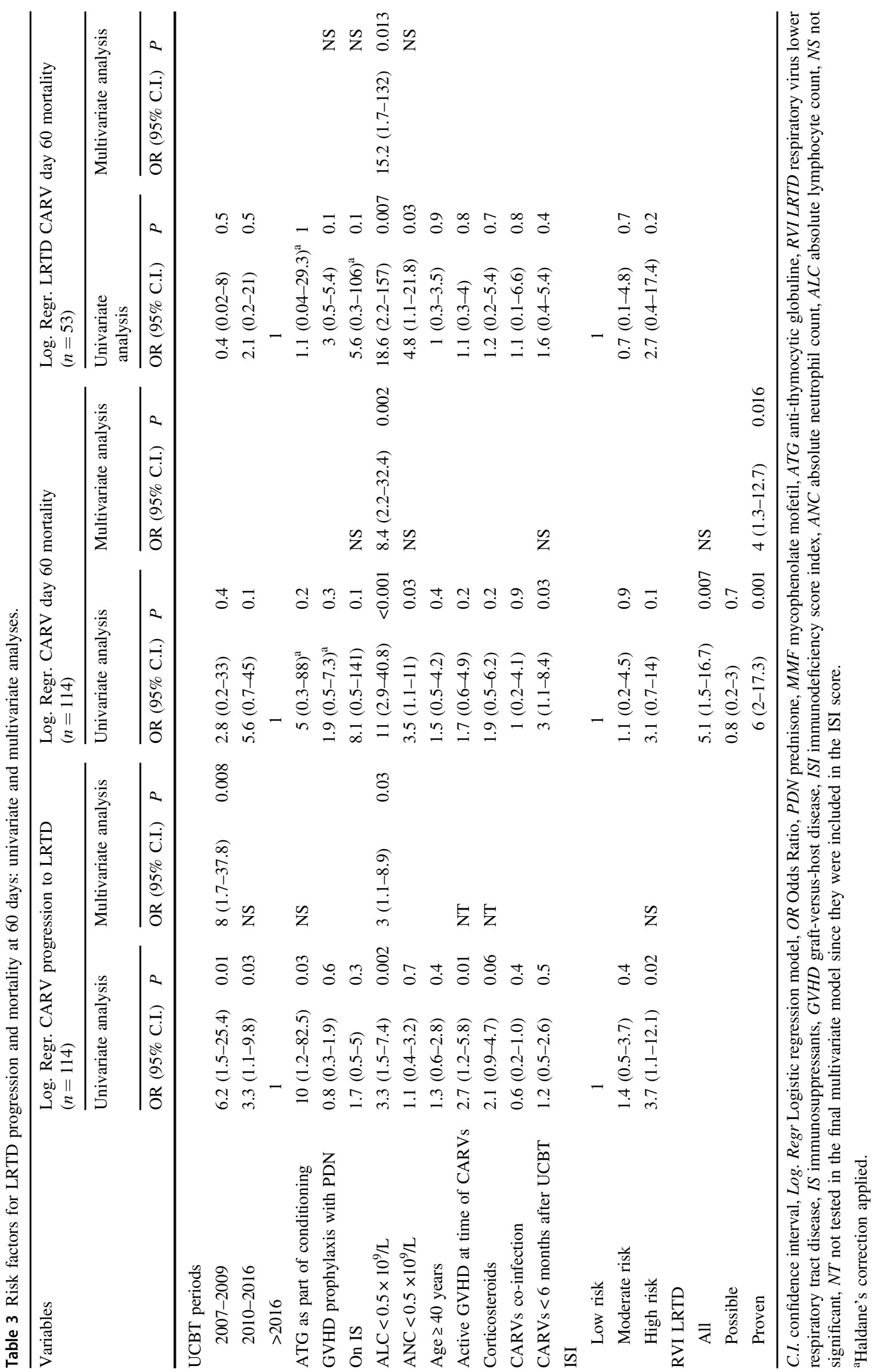



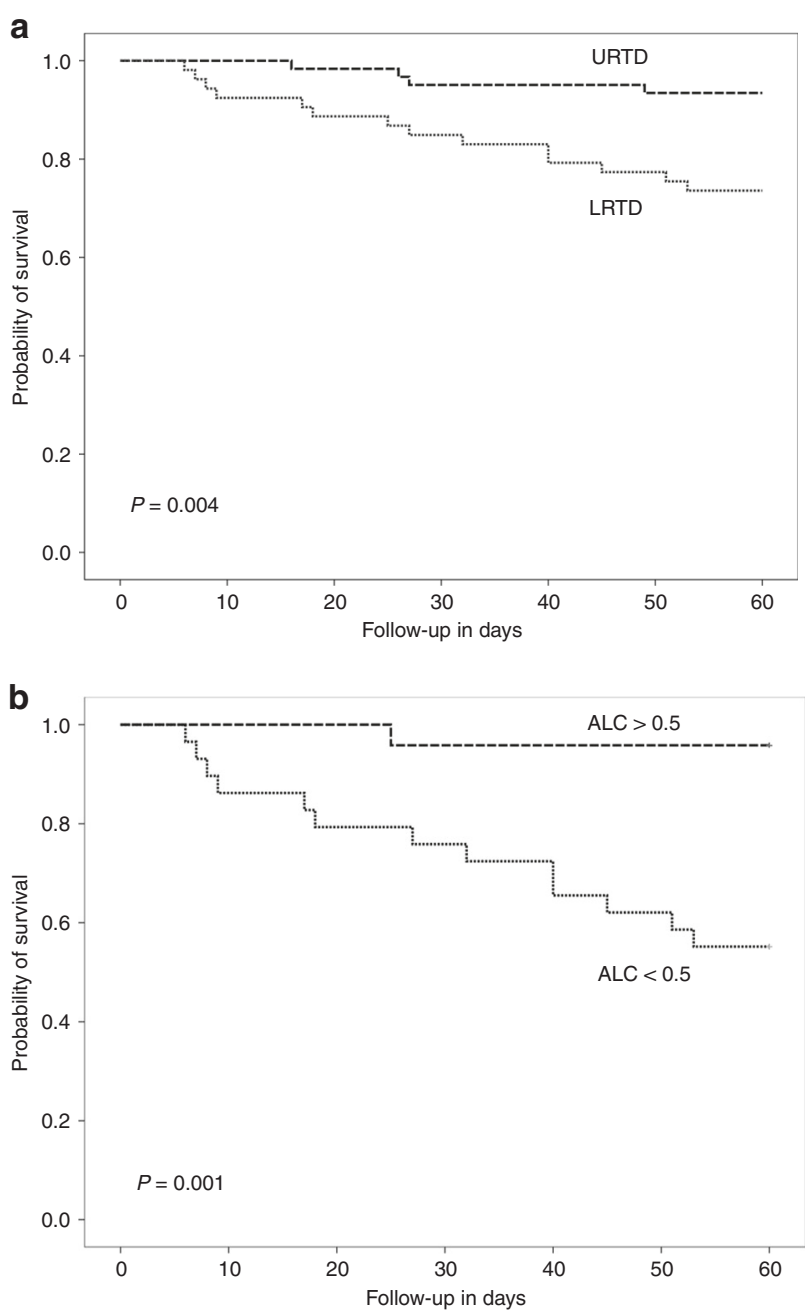

Fig. 3 Survival outcomes. a Probability of survival after URTD and LRTD CARV in the first 60 days. b Probability of survival after LRTD CARV in the first 60 days according to absolute lymphocyte count.

infections are still of major concern in the UCBT setting and require urgent attention and efforts to improve outcomes.

T-cell immune reconstitution is of utmost importance for viral infectious disease and in the UCBT setting T-cell reconstitution is challenging [30]. In this sense, lymphocytopenia was independently associated with both overall CARV infection and CARV LRTD day 60 all-cause mortality. It should be highlighted, that most studies performed to date focusing on immune reconstitution following UCBT for hematological malignancies, have demonstrated extremely severe T-cell lymphopenia that extended all throughout the 1st year [31, 32], as a risk factor for severe disease, which is in accordance with previous observations for other viral infections such as CMV [33]. In our study, mortality from LRTD was relatively low and $16 \%$ died of any cause within 60 days from diagnosis of CARV infection. Usually occurred in the presence of aggressive co-pathogens (10/17 deaths). However, we could not provide evidence in the multivariate analysis that CARV LRTD with bacterial coinfections displayed a negative effect in mortality. In addition, we were not able to provide evidence of the clinical usefulness of the ISI score in predicting mortality in multivariate analysis in the CARV and LRTD CARV cohorts in the setting of UCBT.

We acknowledge that our study has some limitations that include the retrospective design, changes in transplant practices over time and the inclusion of different CARVs screening technics in different periods. However, the singlecenter nature and the inclusion of patients through the era of PCR should be regarded as strength. In addition, our series received a relatively homogeneous conditioning regimens and supportive care, including monitoring, management and supportive care of CARV infections. Our study allowed us to observe a number of potentially therapeutically relevant observations and provide novel data on the occurrence of CARV infections in this setting.

In conclusion, we observed a relatively high incidence of CARV LRTD in recipient undergoing UCBT. CARV infections showed a negative effect on transplant outcomes. The identification of RFs for CARV infections, LRTD progression and mortality provide an awareness of this risk and prevention strategies against LRTD are necessary. These strategies may include an adherence of visitors and staff to hand-washing routines, reducing the exposure of UCBT patients to infected individuals, vaccination, and potentially antiviral prevention strategies in the future.

Author contributions JM, JS, and JLP conceived the study and interpreted the data; JM, and JLP wrote the paper; JM, JS, and JLP, performed the statistical analyses; IL, AB-R, MS, MDG, MG, EMGB, GFS, MAS, reviewed the paper and contributed to the final draft.

\section{Compliance with ethical standards}

Conflict of interest The authors declare that they have no conflict of interest.

Publisher's note Springer Nature remains neutral with regard to jurisdictional claims in published maps and institutional affiliations.

\section{References}

1. Shah JN, Chemaly RF. Management of RSV infections in adult recipients of hematopoietic stem cell transplantation. Blood. 2011;117:2755-63.

2. Renaud C, Campbell AP. Changing epidemiology of respiratory viral infections in hematopoietic cell transplant recipients and solid organ transplant recipients. Curr Opin Infect Dis. 2011;24:333-43.

3. Khanna N, Widmer AF, Decker M, Steffen I, Halter J, Heim D, et al. Respiratory syncytial virus infection in patients with hematological diseases: single-center study and review of the literature. Clin Infect Dis. 2008;46:402-12. 
4. Chemaly RF, Hanmod SS, Rathod DB, Ghantoji SS, Jiang Y, Doshi A, et al. The characteristics and outcomes of parainfluenza virus infections in 200 patients with leukemia or recipients of hematopoietic stem cell transplantation. Blood Am Soc Hematol. 2012;119:2738-45.

5. Ustun C, Slabý J, Shanley RM, Vydra J, Smith AR, Wagner JE, et al. Human parainfluenza virus infection after hematopoietic stem cell transplantation: risk factors, management, mortality, and changes over time. Biol Blood Marrow Transplant. 2012;18:1580-8.

6. Ljungman P, Ward KN, Crooks BN, Parker A, Martino R, Shaw $\mathrm{PJ}$, et al. Respiratory virus infections after stem cell transplantation: a prospective study from the Infectious Diseases Working Party of the European Group for Blood and Marrow Transplantation. Bone Marrow Transplant. 2001;28:479-84.

7. Piñana JL, Gómez MD, Pérez A, Madrid S, Balaguer Rosello A, Giménez E, et al. Community-acquired respiratory virus lower respiratory tract disease in allogeneic stem cell transplantation recipient: risk factors and mortality from pulmonary virusbacterial mixed infections. Transpl Infect Dis. 2018;20:e12926.

8. Piñana JL, Madrid S, Pérez A, Hernández-Boluda JC, Giménez E, Terol MJ, et al. Epidemiologic and clinical characteristics of coronavirus and bocavirus respiratory infections after allogeneic stem cell transplantation: a prospective single-center study. Biol Blood Marrow Transplant. 2018;24:563-70.

9. Chemaly RF, Ghosh S, Bodey GP, Rohatgi N, Safdar A, Keating MJ, et al. Respiratory viral infections in adults with hematologic malignancies and human stem cell transplantation recipients: a retrospective study at a major cancer center. Medicine. 2006;85:278-87.

10. Martino R, Porras RP, Rabella N, Williams JV, Rámila E, Margall $\mathrm{N}$, et al. Prospective study of the incidence, clinical features, and outcome of symptomatic upper and lower respiratory tract infections by respiratory viruses in adult recipients of hematopoietic stem cell transplants for hematologic malignancies. Biol Blood Marrow Transplant. 2005;11:781-96.

11. Sanz J, Boluda JCH, Martin C, González M, Ferrá C, Serrano D, et al. Single-unit umbilical cord blood transplantation from unrelated donors in patients with hematological malignancy using busulfan, thiotepa, fludarabine and ATG as myeloablative conditioning regimen. Bone Marrow Transplant. 2012;47:1287-93.

12. Moscardó F, Sanz J, Carbonell F, Sanz MA, Larrea L, Montesinos P, et al. Effect of CD8 Cell content on umbilical cord blood transplantation in adults with hematological malignancies. Biol Blood Marrow Transplant. 2014;20:1744-50.

13. Piñana JL, Sanz J, Picardi A, Ferrá C, Martino R, Barba P, et al. Umbilical cord blood transplantation from unrelated donors in patients with Philadelphia chromosome-positive acute lymphoblastic leukemia. Haematologica. 2014;99:378-84.

14. Sanz J, Wagner JE, Sanz MA, DeFor T, Montesinos P, Bachanova $\mathrm{V}$, et al. Myeloablative cord blood transplantation in adults with acute leukemia: comparison of two different transplant platforms. Biol Blood Marrow Transplant. 2013;19:1725-30.

15. Piñana JL, Hernández-Boluda JC, Calabuig M, Ballester I, Marín $\mathrm{M}$, Madrid S, et al. A risk-adapted approach to treating respiratory syncytial virus and human parainfluenza virus in allogeneic stem cell transplantation recipients with oral ribavirin therapy: a pilot study. Transpl Infect Dis. 2017;19.

16. Hirsch HH, Martino R, Ward KN, Boeckh M, Einsele H, Ljungman P. Fourth European Conference on Infections in Leukaemia (ECIL-4): guidelines for diagnosis and treatment of human respiratory syncytial virus, parainfluenza virus, metapneumovirus, rhinovirus, and coronavirus. Clin Infect Dis. 2013;56:258-66.
17. Piñana JL, Pérez A, Montoro J, Giménez E, Dolores Gómez M, Lorenzo I, et al. Clinical effectiveness of influenza vaccination after allogeneic hematopoietic stem cell transplantation: a crosssectional prospective observational study. Clin Infect Dis. 2019;68:1894-903.

18. Seo S, Xie H, Campbell AP, Kuypers JM, Leisenring WM, Englund JA, et al. Parainfluenza virus lower respiratory tract disease after hematopoietic cell transplant: viral detection in the lung predicts outcome. Clin Infect Dis. 2014;58:1357-68.

19. Gooley TA, Leisenring W, Crowley J, Storer BE. Estimation of failure probabilities in the presence of competing risks: new representations of old estimators. Stat Med. 1999;18:695-706.

20. Fine JP, Gray RJ. A proportional hazards model for the subdistribution of a competing risk. $\mathrm{J}$ Am Stat Assoc. 2012;94:496-509.

21. Gray RJ. A class of K-sample tests for comparing the cumulative incidence of a competing risk. Ann Stat. 1988;16:1141-54.

22. HALDANE JB. The estimation and significance of the logarithm of a ratio of frequencies. Ann Hum Genet. 1956;20:309-11.

23. Kaplan EL, Meier P. Nonparametric estimation from incomplete observations. J Am Stat Assoc. 1958;53:457-81.

24. Milano F, Campbell AP, Guthrie KA, Kuypers J, Englund JA, Corey $\mathrm{L}$, et al. Human rhinovirus and coronavirus detection among allogeneic hematopoietic stem cell transplantation recipients. Blood Am Soc Hematol. 2010;115:2088-94.

25. Hassan IA, Chopra R, Swindell R, Mutton KJ. Respiratory viral infections after bone marrow/peripheral stem-cell transplantation: the Christie hospital experience. Bone Marrow Transplant. 2003;32:73-7.

26. Ambrosioni J, Bridevaux PO, Wagner G, Mamin A, Kaiser L. Epidemiology of viral respiratory infections in a tertiary care centre in the era of molecular diagnosis, Geneva, Switzerland, 2011-2012. Clin Microbiol Infect. 2014;20:O578-84.

27. García-Cadenas I, Rivera I, Martino R, Esquirol A, Barba P, Novelli $\mathrm{S}$, et al. Patterns of infection and infection-related mortality in patients with steroid-refractory acute graft versus host disease. Bone Marrow Transplant. 2017;52:107-13.

28. Laughlin MJ, Eapen M, Rubinstein P, Wagner JE, Zhang M-J, Champlin RE, et al. Outcomes after transplantation of cord blood or bone marrow from unrelated donors in adults with leukemia. $\mathrm{N}$ Engl J Med. 2004;351:2265-75.

29. Sanz J, Cano I, González-Barberá EM, Arango M, Reyes J, Montesinos $\mathrm{P}$, et al. Bloodstream infections in adult patients undergoing cord blood transplantation from unrelated donors after myeloablative conditioning regimen. Biol Blood Marrow Transplant. 2015;21:755-60.

30. Politikos I, Boussiotis VA. The role of the thymus in T-cell immune reconstitution after umbilical cord blood transplantation. Blood. 2014;124:3201-11.

31. Komanduri KV, St John LS, de Lima M, McMannis J, Rosinski S, McNiece I, et al. Delayed immune reconstitution after cord blood transplantation is characterized by impaired thymopoiesis and late memory T-cell skewing. Blood. 2007;110:4543-51.

32. Szabolcs P, Cairo MS. Unrelated umbilical cord blood transplantation and immune reconstitution. Semin Hematol. 2010;47:22-36.

33. Einsele H, Ehninger G, Steidle M, Fischer I, Bihler S, Gerneth F, et al. Lymphocytopenia as an unfavorable prognostic factor in patients with cytomegalovirus infection after bone marrow transplantation. Blood 1993;82:1672-8. 